\title{
Based on network pharmacology and molecular docking to explore the mechanism of Achyranthis bidentate radix in the treatment of Renal cell carcinoma
}

Yu Liu

Hospital of Chengdu University of Traditional Chinese Medicine

Mingquan Li (D1563540954@qq.com)

Hospital of Chengdu University of Traditional Chinese Medicine https://orcid.org/0000-0001-94024406

\section{LaiKuan Teh}

Universiti Tunku Abdul Rahman

\section{Liangbin zhao}

Hospital of Chengdu University of Traditional Chinese Medicine

Naijing Ye

Hospital of Chengdu University of Traditional Chinese Medicine

Bo Qu

Hospital of Chengdu University of Traditional Chinese Medicine

\section{Hongmei Lu}

Hospital of Chengdu University of Traditional Chinese Medicine

\section{Ling Wu}

Hospital of Chengdu University of Traditional Chinese Medicine

Lihua Wu

Hospital of Chengdu University of Traditional Chinese Medicine

Anqi Tang

Hospital of Chengdu University of Traditional Chinese Medicine

\section{Research}

Keywords: Achyranthis bidentate radix, Renal cell carcinoma, network pharmacology

Posted Date: March 24th, 2021

DOl: https://doi.org/10.21203/rs.3.rs-339388/v1 
License: (c) (i) This work is licensed under a Creative Commons Attribution 4.0 International License. Read Full License 


\section{Abstract}

Background: Renal cell carcinoma (RCC) is a common malignant tumor of the urinary system. The incidence rate was increasing year by year, which has become a worldwide health problem. Achyranthis bidentate radix $\mathbb{A B R}$ ) is a traditional Chinese medicine that has been commonly reported on the prevention and treatment of RCC. However, the detailed mechanism of ABR is unclear. Therefore, this study aims to explore the possible molecular mechanism of ABR in the treatment of RCC based on network pharmacology and molecular docking.

Methods: The main active compounds of ABR were obtained from pharmacology analysis platform of the Chinese Medicine System (TCMSP). The targets of ABR active compounds were obtained from TCMSP and Swiss Target Prediction. The targets of RCC were then screened in the Gene Cards and Online Mendelian Inheritance in Man (OMIM). The network of "medicine-ingredients-disease-targets" was established employing network pharmacology, and the key targets and core pathways were determined by $\mathrm{R}$ analysis. The molecular docking method was used to evaluate the binding activity between the target and the active compounds of ABR.

Results: Twenty active compounds were found from ABR and are involving in 164 targets. The results of network analysis indicated that the targets mainly involve biological processes such as active oxygen metabolism, oxidative stress, cell proliferation, and apoptosis. ABR was able to treat RCC through regulating AGE-RAGE, PI3K-Akt, MAPK, JAK-STAT, NF-KB, and other major signaling pathways. Quercetin, baicalein, kaempferol had a strong binding to the target proteins of and MAPK1, AKT1, HSP90AA1.

Conclusion: Flavonoids in ABR may be the material basis for its treatment of RCC. The mechanisms of action

were through regulating active oxygen metabolism, inhibiting oxidative stress, cell proliferation, activating tumor cell apoptosis pathway, regulating protein kinase activity and nuclear receptor Activity, etc.

\section{Introduction}

RCC is a malignant tumor originating from renal tubular epithelium, and its incidence is increasing year by year [1]. Studies have shown that its incidence is closely related to exposure to nephrotoxic industrial chemicals, smoking, obesity, and genetic susceptibility [2]. Early renal cell carcinoma usually has no obvious clinical symptoms [3]. Therefore, early diagnosis and treatment for renal cell carcinoma have become a major problem. At present, surgery is the preferred treatment for renal cell carcinoma [4]. However, most patients have lost the opportunity for surgical treatment when the disease was diagnosed. Meanwhile, renal cell carcinoma is highly resistant to radiotherapy and chemotherapy [5], cytokines, and immunity treatment. The effect of interference treatment is also poor [6]. Although the utilization of molecularly targeted drugs such as PD-1 and PDL-1 inhibitors have achieved good effects in clinical applications, the adverse reactions are also obvious [7]. In recent years, Traditional Chinese Medicine has been widely used in the treatment of tumors. Traditional Chinese medicine has been recognized by 
clinicians and patients because of its ability to alleviate the side effects of targeted drugs and biological immunotherapy; improve the quality of life of patients, and prolong the survival time of patients [8].

ABR was first published in "Shen Nong's Materia Medica". The taste is bitter, sweet, sour, and smooth, and it belongs to the liver and kidney channels. It is good at promoting blood circulation, invigorating the liver and kidney, strengthening the bones and muscles, removing water and drenching, igniting fire (water) down. It can be used to treat amenorrhea caused by blood stasis, dysmenorrhea, ache on waist and knees, and lower extremity weakness[9]. Modern studies have shown that the ginsenoside (Ro) in ABR can inhibit the invasion and metastasis of tumor cells and the adhesion to human endothelial cells by specifically inhibiting the expression of integrins av $\beta 6$, MMP-2, and MMP-9 [10]. Li et al. proposed that the polysaccharide in ABR has many functions such as anti-tumor, anti-oxidation, strengthening immunity, and regulating the intestinal microenvironment [11].

Due to the multi-component, multi-target and multi-path characteristics of traditional Chinese medicine, the substance basis and mechanism of action of ABR are difficult to clarify. Identification of the mechanism of action through network pharmacology has been proposed by Hopkins, a British pharmacologist to study the molecular mechanism of drug intervention in diseases from multidimensional perspectives such as systems biology and multidirectional pharmacology. Many studies have shown that studying the mechanism of traditional Chinese medicine through network pharmacology is accurate and reliable. The current study was utilizing network pharmacological methods to construct a medicine-ingredients-disease-targets network. Network topology analysis was performed to explore the multi-component, multi-target, multi-path mechanism of action of 20 active compounds from $A B R$ for the treatment of renal cell carcinoma. This would contribute the fundamentals information on the mechanism of treatment of ABR on RCC[12].

\section{Methods}

\subsection{Acquisition of chemical compounds and screening of active compounds in ABR}

Through Traditional Chinese Medicine Systems Pharmacology Database and Analysis Platform (TCMSP) (http://Isp.nwsuaf.edu.cn/tcmsp.php), "Achyranthis bidentate radix" was used as the keyword to identify for all the chemical compounds that present in ABR. An oral availability (OB) greater than $30 \%$ and a pharmacokinetic value (DL) greater than 0.18 were used as the criteria to screen for the active compounds in ABR.

\subsection{Target prediction of the active compounds}

In silico predictions of the target for the active compounds from ABR were queried by using the TCMSP and SwissTargetPrediction(http://www.swisstargetprediction.ch/). The target proteins were identified for the respective gene using Perl (http://www.perl.org/) and the UniProt database (http://www.UniProt.org/).

\subsection{Target prediction of disease}


To reveal the target genes related to RCC, "renal cell carcinoma" was used as the keyword in databases such as Genecards (tps://w ww.genecards.org/) Online Mendelian Inheritance in Man(OMIM) (http://ci.smu.edu.cn/ CooLGeN /). The genes target RCC that with the top $10 \%$ of the score were retrieved and the duplicate targets were removed from the results.

\subsection{Intersection of active compounds with RCC}

R package (https://www.rproject.org/) was downloaded and the command code was entered to install the toolkit for drawing a Venn diagram. Then, the list of active compounds from ABR and the target gene of RCC was entered into the R package using specific code to generate a Venn diagram and the "ingredients to disease" list to describe the intersection between the compounds with RCC.

\subsection{Network construction and analysis}

Network construction was generated by importing the "ingredients to disease" list into Cytoscape 3.7.1 software with ABR compounds name and disease name. The nodes of the "medicine-ingredients-diseasetargets (M-I-D-T)" network were used to represent target proteins and edges were used to represent the relationships between active compounds, disease, and target proteins.

\subsection{Construction of the protein interaction network}

To construct the protein interaction network, the "ingredients to disease" list was imported into the Search Tool for the Retrieval of Interacting Genes (STRING) database (http://string-db.org) with "Homo sapiens" as species and the minimum interaction threshold adjusted to 0.9 . This is to determine the relationships between potential targets of ABR in the treatment of RCC. The interactive network was drawn with the imported node 1, node 2, and the combined score information using Cytoscape 3.7.1 software. The node size was set to reflect the size of the degree. The thickness of the edge was to reflect the size of the score to obtain the final protein interaction network. R package was then used to screen the hub proteins for the number of junction nodes between all proteins and the top 30 proteins.

\subsection{Gene ontology and pathway enrichment analysis}

The enrichment analysis of Gene Ontology (GO) functions and Kyoto Encyclopedia of Genes and Genomes (KEGG) pathways were carried out to construct the network between the active compounds with the disease target gene. The results were obtained at P-adjusted value $(p<0.05)$.

\subsection{Molecular docking}

To perform molecular docking, the structure of related proteins was downloaded from the RCSB database (https://www.rcsb.org/) and solvent molecules and ligands were removed using Pymol software whilst AutoDock software was used to perform hydrogenation, electron addition and ROOT operation. The chemical structure of active compounds was downloaded from Pubchem data (https://pubchem.ncbi.nlm.nih.gov/). After the completion of the molecular docking, the protein structure 
is set as a rigid macromolecule, and the algorithm was obtained as Local Search Parameters. Pymol software was used to map the result of the lowest binding energy between each target and the active compounds.

\section{Results}

\subsection{Active compounds from ABR}

From TCMSP database, one hundred and seventy-six (176) chemical compounds were found from Achyranthis bidentate radix. Among this, twenty (20) active compounds were identified as shown in Table1.

\subsection{Potential targets of the active compounds and RCC}

From TCMSP and SwissTargetPrediction, a total of 1140 potential targets of ABR active ingredients were identified. Meanwhile, from the databases of disease genes, there was a total of 1046 potential targets were successfully retrievedwith top $10 \%$ of the score and removal of duplicate targets. All these potentialtargets were found closely related with the occurrence and development of RCC.

\subsection{Intersection of active compound with disease}

Intersection of active compound and disease target was determined using R platform. The Venn diagram showed 164 potential targets had relationships with active compounds and RCC. (Figure 1). Figure 2 presents the medicine-ingredients-targets-disease (MITD) network which demonstrating the relationship between ABR and RCC.

\subsection{Protein-protein interaction (PPI) network}

From the PPI network, both RCC targets and active ingredient potential targets presented 164 duplicate genes. These duplicate genes could potentially become targets for thetreatment of RCC. PPI network analysis of the potential target groups was then carried out to study the interaction of the targets in vivo and to search for the hub genes. The node1, node2 and combined score informationin the exported file were imported into Cytoscape 3.7.1 software. The node sizewas adjusted to reflect the size of the degree, and the thicknessof the edge to reflect the size of the score to obtain the final PPI network (Figure 3). The top 30 genes that had a close relationship with other genes were represented viaa bar plot that clearly described these 30 gene targets in terms of their key positions in the PPI network. The top five genes were MAPK1(Mitogen-activatedprotein kinase 1),STAT3(Signal transducer and activator of transcription 3),AKT1(RAC-alpha serine/threonine-protein kinase),HSP90AA1(Heat shock protein HSP90alpha),GRB2(Growth factor receptor-boundprotein 2)(Figure 4).

\subsection{Gene ontology (GO) and The Kyoto Encyclopedia of Genes and Genomes (KEGGpathway enrichment analysis}


To illustrate the mechanism underlying the effects of ABR active ingredients on RCC more comprehensively and concretely, GO enrichment analysis of the 164 common targets were performed. The analysis results indicated that $A B R$ acted on RCC through regulating multiple biological processes (BP) $(p<0.05)$ (Figure 5) consisting of response to oxidative stress (G0:0006979), cellular response to oxidative stress (G0:0034599),regulation of reactive oxygen species metabolic process (G0:2000377), epithelial cellproliferation (GO:0050673), and extrinsic apoptotic signaling pathway (GO:0097191).The main cellular components $(\mathrm{CC})$ terms $(p<0.05)$ (Figure 6) were membrane raft (G0:0045121), membrane microdomain (GO:0098857), focal adhesion (G0:0005925), cell-substrate adherens junction (G0:0005924) and cell-substrate junction (G0:0030055), while the molecular functions (MF) terms ( $p<$ 0.05) (Figure 7) included protein tyrosine kinase activity (GO:0004713),transmembrane receptor protein tyrosine kinase activity(G0:0004714), transmembrane receptor protein kinase activity (G0:0019199), phosphatase binding (GO:0019902) and nuclear receptor activity (GO:0004879). Figure 5 to 7 presents the gene ratio of the number of target genes involved in one biological processto the number of all annotated genes. Higher ratio indicating the higher level of enrichment. The size of the dot reflects the number of target genes in the analysis, and the different colors of the dots indicate different $P$-adjusted value ranges.

To elucidate the critical pathways among the 164 potential targets in terms of RCC therapy, the top 20 pathways were filtered according to the $P$-adjusted value $<0.05$ (Figure8). The pathways involved were found in AGE- RAGE signaling pathway in diabetic complications (hsa04933), and prostate can cer (hsa05215), EGFR tyrosine kinase inhibitor resistance (hsa01521), PI3K-Akt si gnaling pathway (hsa04151), Karposi sarcoma-associated herpes virus infection (hsa 04151),Hepatitis B (hsa05161), Pancreatic cancer (hsa05212), Bladder cancer (hsa052 19), Human cytomegalovirus infection (hsa05163), Non-small cell lung cancer (hsa0 5223), Proteoglycans in cancer(hsa05205), Fluid shear stress and atherosclerosis (hsa05418), MAPK signaling pathway (hsa04010), Hepatitis C (hsa05160), Endocrine resistance(hsa01522), Colorectal cancer (hsa05210), TNF signaling pathway (hsa04668), Measles (hsa05162), Hepatocellular carcinoma (hsa05225), Epstein-Barr virus infection (hsa05169). Analytical method using GO analysis presented same pathways involved as in the GO dot plot (Figure9).

\subsection{Molecular docking}

Three key active compounds showed the lowest binding energies $(<0)$ with the targets of MAPK1, AKT1, HSP90AA1 (Table2). The energies were found less than $-5.0 \mathrm{~kJ} \cdot \mathrm{mol}^{-1}$, indicating that the three key pharmacodynamic components bind well with the targets MAPK1, AKT1, and HSP90AA1. Table 2 present the binding docking energy. Wogonin was found had the lowest binding energy with the target AKT1. This indicated that wogonin binds better to AKT1 as target. In addition, the binding ability of kaempferol and quercetin to MAPK1 as target was better than that to AKT1 and HSP90AA1. The docking pattern is presented in Figure 1, where the structure of the compound is represented by sticks, different branches of the protein are represented by different colors, and the yellow dashed line represents its hydrogen bond, marking the position of the hydrogen bond and the compound in the compound. The number of hydrogen 
bonds between wogonin and the target AKT1 was at 1, and the number of hydrogen bonds between kaempferol and quercetin and the target MAPK1 were 3 and 2 respectively.

\section{Discussion}

This study demonstrated twenty candidate active compounds of $A B R$ with $O B \geq 30 \%$ and $D L \geq 0.18$ when screened using the TCMSP database. A total of 164 were found intersect between the target of ABR and the target of RCC. From the ABR medicine-ingredients-disease-targets network diagram (Figure2), one compounds were found able to act on multiple targets, and each target were found corresponding to multiple components, This was fully reflecting the multi-component and multi-target of traditional Chinese medicine Point characteristics. Active compounds from ABR with the highest median values were quercetin, wogonin and kaempferol. All these were were natural flavonoids that naturally present in ABR [13].

Modern studies have shown that quercetin has anti-tumor, anti-inflammatory and anti-oxidant effects. In vivo and in vitro experiments had elucidated that quercetin able to inhibit cell proliferation, promote cell apoptosis, inhibit angiogenesis and regulate cell cycle. Quercetin exhibit autophagy ability and thus exerts an anti-tumor effect whilst suppress tumor progression by regulating epigenetics. While regulating of epigenetics presented anti-tumor effect by regulating the expression of miRNA and the level of DNA methylation, and enhancing the sensitivity of tumor cells to chemotherapy[14].Wogonin has antitumor,anti-inflammatory,anti-viral,anti-oxidation and other pharmacological effects. Wogonin can induce apoptosis in vitro. Wogonin eliminates the oxidized free radicals by blocking the cell cycle, inducing apoptosis, inhibiting the growth, invasion and metastasis of tumor cells, enhancing the sensitivity of chemotherapeutic drugs, triggering the death of autophagic cells, etc[15].Kaempferol was commonly found in majority of traditional Chinese medicines. It exhibits anti-tumor, anti-inflammatory, and antioxidant effects. Its anti-tumor effects was involving inflammation, apoptosis and angiogenesis, metastasis. Kaempferol can selectively inhibit cancer cells without affecting normal cells. Nanoparticles utilizing kaempferol provide a new direction for tumor treatment [16].

According to earlier studies,overexpression of PTGS2 can promote tumor formation, invasion and metastasis. This can be explained as this will increase cell proliferation, inhibit apoptosis, enhance invasiveness, induce immune response and chronic activation of angiogenesis. Studies have shown that PTGS2 was significantly expressed in Renal tubular epithelioma and RCC. Overexpression of PTGS2 was found positively correlated with tumor size,grade, and RCC stage in which $63.6 \%$ of RCC patients have high expression [17]. Ting et al. found that ESR1 was associated with the occurrence of RCC by mining the GEO database [18]. Studies have shown that high expression of PTGS1 is related to tumor size, TNM stage, pathological stage, and tumor recurrence. Patients with high expression of PTGS1 have shorter survival times [19]. From this, we speculate that quercetin and kaempferol from ABR may act on key targets such as PTGS2, ESR1, and PTGS1. These active compounds play an important role in the pharmacology of ABR. 
The results of molecular docking showed that MAPK1, AKT1, HSP90AA1 have high binding ability to the core compounds in Renal tubular epithelioma. This speculated that the core compounds in ABR may treat RCC by regulating MAPK1, AKT1, HSP90AA1. MAPK1 is a key target in promoting nuclear accumulation and transcriptional activity of HIF-1a.High MAPK1 activity was found in advanced RCC and its activity level can predict the occurrence of local disease metastasis [20]. AKT1 is a serine/threonine kinase activated downstream of phosphatidylinositol 3 kinase (PI3K) and mediates various cell survival signals through mTOR. Increased expression of AKT1 is closely related to the tumor grade and metastasis of kidney cancer [21]. HSP90AA1 is a subtype of Hsp90, which is highly expressed in cancer. Its expression level is closely related to the disease progression and survival of cancer patients [22].

Gene Ontology (GO) functional analysis indicates that ABR treatment of RCC may be achieved by regulating reactive oxygen species metabolism, inhibiting oxidative stress, cell proliferation, activating tumor cell apoptosis pathways, regulating protein kinase activity, nuclear receptor activity and other processes. When the level of reactive oxygen species (ROS) in the body increases, it will subsequently promote and maintains the signal transduction of tumorigenic cells in leading to tumor cell proliferation, survival, autophagy and metastasis [23].Nuclear receptors (NRs) are ligand-activated transcription factors involved in biological functions such as cell differentiation, metabolism, inflammation, and tumors. Therefore, from autoimmunity to malignant tumors, dysregulation of the role of nuclear receptors plays a vital role [24]. KEGG pathway analysis results showed that the AGE-RAGE signaling pathway among diabetic complications is the most significant. This pathway includes PI3K-Akt signaling pathway, MAPK signaling pathway, JAK-STAT signaling pathway, nuclear transcription factor-KB and other major signals. These pathways are closely related to RCC.PI3K-Akt signaling pathway can regulate the basic functions of cells, such as transcription, translation, proliferation, growth and survival. Compared with other signaling pathways, it has gene targeting in more pathway components and tumor types, providing many potential targets for tumor treatment.The PI3K-Akt signaling pathway is moderately mutated and highly activated in RCC, becoming a promising drug target for RCC[25].MAPK signaling pathway plays an important role in the process of normal cell proliferation and differentiation. Earlies findings have shown that activation of MAPK signaling pathway is involved in the occurrence, metastasis and angiogenesis of various tumors including RCC. Blocking one or more MAPK signaling pathways can destroy the RCC vasculature, thereby achieving tumor suppression [26]. The JAK-STAT pathway is the main and classic pathway of interferon signaling. Studies have shown that the expression of JAK1 and STAT1 in RCC tissue is significantly lower than that of normal kidney tissue [27]. The low expression of the above two key molecules may prevent JAK-STAT signaling pathway from being activated or not fully activated, which in turn affects the transcription of related genes downstream affects the anti-tumor biological effects of interferon[28].NF-кB,a classical signaling pathway related to inflammation, is closely related to RCC.RCC grade, invasion and metastasis are directly related to the expression and activation of NF- $\mathrm{B}$ [29].Besides, PD-L1 expression and PD-1 checkpoint pathway in cancer [30], HIF-1 signaling pathway and other signaling pathways are also related to the occurrence of RCC[31].

\section{Conclusion}


In this study, based on network pharmacology and molecular docking, we performed target prediction and pathway analysis on the active compounds of $A B R$, and explored the potential mechanism of action of $A B R$ in the treatment of RCC from multiple angles.Inhibition of oxidative stress, cell proliferation, activation of apoptotic pathways, regulation of protein kinase activity, nuclear receptor activity, and other mechanisms play an anti-renal cell carcinoma role, providing a basis and direction for the screening of evaluation indicators for subsequent clinical trials. As current network pharmacology study is solely based on bioinformatics tools, thus further screening and validation in vivo and in vitro should be carried out to elucidate the pharmacology of ABR in treating RCC.

\section{List Of Abbreviations}

$\mathrm{ABR} \unrhd$ Achyranthis bidentate radix

RCC『Renal cell carcinoma

\section{Declarations}

\section{Ethics approval and consent to participate}

Not applicable

\section{Consent for publication}

manuscript is approved by all authors for publication

\section{Availability of data and materials}

The dataset(s) supporting the conclusions of this article are available in the TCMSP \http://Isp.nwsuaf.edu.cn/tcmsp.php囚,SwissTargetPrediction(http://www.swisstargetprediction.ch/) खPerl

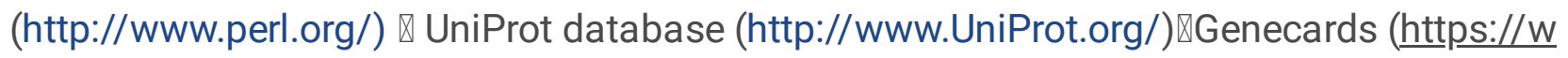
ww.genecards.org/) $₫ \mathrm{OMIM}(\mathrm{http} / / /$ ci.smu.edu.cn/ CooLGeN/)『R package (https://www.rproject.org/) and STRING database (http://string-db.org).

\section{Competing interests}

None.

\section{Funding}

There is no Funding support

\section{Authors' contributions}


Thanks to Professor Mingquan Li for providing research ideas,Liangbin Zhao ,Naijing Ye and Bo Qu for technical support.,Hongmei Lu , Ling Wu , Lihua Wu's, Anqi Tang's help in data collection囚Help with language editing by Lai Kuan teh.

\section{Acknowledgements}

Not applicable

\section{References}

[1]Sanchez DJ, Simon MC. Genetic and metabolic hallmarks of clear cell renal cell carcinoma.Biochim Biophys Acta Rev Cancer. 2018;1870(1):23-31.

[2]Petejova N, Martinek A. Renal cell carcinoma: Review of etiology, pathophysiology and risk factors. Biomed Pap Med Fac Univ Palacky Olomouc Czech Repub. 2016;160(2):183-194.

[3]Petejova N, Martinek A.Renal cell carcinoma: Review of etiology, pathophysiology and risk factors. Biomed Pap Med Fac Univ Palacky Olomouc Czech Repub. 2016 Jun;160(2):183-94.

[4]Roos FC, Rübben H, Stief C, Stöckle M, Thüroff JW. Operative Therapie des Nierenzellkarzinoms .Surgical treatment for renal cell carcinoma. Aktuelle Urol. 2010;41(4):252-256.

[5]Posadas EM, Limvorasak S, Figlin RA. Targeted therapies for renal cell carcinoma. Nat Rev Nephrol. 2017;13(8):496-511.

[6] Heicappell R, Ackermann R. Rationale for immunotherapy of renal cell carcinoma. Urol Res. 1990;18(6):357-372.

[7]Massari F, Santoni M, Ciccarese C, et al. PD-1 blockade therapy in renal cell carcinoma: current studies and future promises. Cancer Treat Rev.

2015;41(2):114-121.

[8]Xiang Y, Guo Z, Zhu P, Chen J, Huang Y. Traditional Chinese medicine as a cancer treatment: Modern perspectives of ancient but advanced science. Cancer Med. 2019 May;8(5):1958-1975.

[9]Wu J, Li J, Li W, Sun B, Xie J, Cheng W, Zhang Q. Achyranthis bidentatae radix enhanced articular distribution and anti-inflammatory effect of berberine in Sanmiao Wan using an acute gouty arthritis rat model. J Ethnopharmacol. 2018 Jul 15;221:100-108.

[10]Jiang Z, Qian J, Dong H, et al. The traditional Chinese medicine Achyranthes bidentata and our de novo conception of its metastatic chemoprevention: from phytochemistry to pharmacology. Sci Rep. 2017;7(1):3888. 
[11]Li N, Yu X, Yu QH, Wang M.Research Progress on Stability of Polysaccharides in Traditional Chinese Medicine Zhongguo Zhong Yao Za Zhi. 2019;44(22):4793-4799.

[12] Li H, Li B, Zheng Y. Exploring the Mechanism of Action Compound-Xueshuantong Capsule in Diabetic Retinopathy Treatment Based on Network Pharmacology. Evid Based Complement Alternat Med. 2020 Sep 9;2020:8467046.

[13]Serafini M, Peluso I, Raguzzini A. Flavonoids as anti-inflammatory agents. Proc Nutr Soc. 2010 Aug;69(3):273-8.

[14]Tang SM, Deng XT, Zhou J, Li QP, Ge XX, Miao L.Pharmacological basis and new insights of quercetin action in respect to its anti-cancer effects. Biomed Pharmacother. 2020;121:109604.

[15]Liu H, Dong Y, Gao Y, et al. The Fascinating Effects of Baicalein on Cancer: A Review. Int J Mol Sci. 2016;17(10):1681.

[16]Imran M, Salehi B, Sharifi-Rad J, et al. Kaempferol: A Key Emphasis to Its Anticancer Potential. Molecules. 2019;24(12):2277.

[17]Tabriz HM, Mirzaalizadeh M, Gooran S, Niki F, Jabri M. COX-2 Expression in Renal Cell Carcinoma and Correlations with Tumor Grade, Stage and Patient Prognosis. Asian Pac J Cancer Prev. 2016;17(2):535538.

[18]Yao T, Wang Q, Zhang W, Bian A, Zhang J. Identification of genes associated with renal cell carcinoma using gene expression profiling analysis. Oncol Lett. 2016;12(1):73-78.

[19]Yu ZH, Zhang Q, Wang YD, et al. Overexpression of cyclooxygenase-1 correlates with poor prognosis in renal cell carcinoma. Asian Pac J Cancer Prev.

2013;14(6):3729-3734.

[20]Wei H, Ke HL, Lin J, Shete S, Wood CG, Hildebrandt MA. MicroRNA target site polymorphisms in the VHL-HIF1a pathway predict renal cell carcinoma risk. Mol Carcinog. 2014;53(1):1-7.

[21]Chu D, Paoletti C, Gersch C, et al. ESR1 Mutations in Circulating Plasma Tumor DNA from Metastatic Breast Cancer Patients. Clin Cancer Res. 2016;22(4):993-999.

[22]Song KH, Oh SJ, Kim S, et al. HSP90A inhibition promotes anti-tumor immunity by reversing multimodal resistance and stem-like property of immune-refractory tumors. Nat Commun. 2020;11(1):562.

[23]Liao Z, Chua D, Tan NS. Reactive oxygen species: a volatile driver of field cancerization and metastasis. Mol Cancer. 2019;18(1):65.

[24]Cathcart P, Lucchesi W, Ottaviani S, et al. Noncoding RNAs and the control of signalling via nuclear receptor regulation in health and disease. Best Pract Res Clin Endocrinol Metab. 2015;29(4):529-543. 
[25]Guo H, German P, Bai S, et al. The PI3K/AKT Pathway and Renal Cell Carcinoma. J Genet Genomics. 2015;42(7):343-353.

[26]Huang D, Ding Y, Luo WM, et al. Inhibition of MAPK kinase signaling pathways suppressed renal cell carcinoma growth and angiogenesis in vivo. Cancer Res. 2008;68(1):81-88.

[27]Li W, Wei Q, Liang J. Phosphorylated signal transducer and activator of transcription 1 is a potential predictor of interferon response in patients with advanced renal cell carcinoma. Mol Med Rep. 2014;9(5):1929-1934.

[28]Shang D, Liu Y, Ito N, Kamoto T, Ogawa O. Defective Jak-Stat activation in renal cell carcinoma is associated with interferon-alpha resistance. Cancer Sci. 2007;98(8):1259-1264.

[29]Morais C, Gobe G, Johnson DW, Healy $H$. The emerging role of nuclear factor kappa B in renal cell carcinoma. Int J Biochem Cell Biol. 2011;43(11):1537-1549.

[30]Kammerer-Jacquet SF, Deleuze A, Saout J, et al. Targeting the PD-1/PD-L1 Pathway in Renal Cell Carcinoma. Int J Mol Sci. 2019;20(7):1692.

[31]Sellitti DF, Doi SQ. MicroRNAs in Renal Cell Carcinoma. Microrna. 2015;4(1):26-35.

\section{Tables}

Table 1 Active compounds from Achyranthis bidentate radix 


\begin{tabular}{llll}
\hline Mol ID & Molecule Name & OB & DL \\
\hline MOL001006 & poriferasta-7,22E-dien-3beta-ol & 42.98 & 0.76 \\
MOL012461 & 28-norolean-17-en-3-ol & 35.93 & 0.78 \\
MOL012537 & Spinoside A & 41.75 & 0.4 \\
MOL001454 & berberine & 36.86 & 0.78 \\
MOL001458 & coptisine & 30.67 & 0.86 \\
MOL000173 & wogonin & 30.68 & 0.23 \\
MOL002643 & Delta7-stigmastenol & 37.42 & 0.75 \\
MOL002714 & baicalein & 33.52 & 0.21 \\
MOL002776 & Baicalin & 40.12 & 0.75 \\
MOL002897 & epiberberine & 43.09 & 0.78 \\
MOL000358 & beta-sitosterol & 36.91 & 0.75 \\
MOL003847 & Inophyllum E & 38.81 & 0.85 \\
MOL000422 & kaempferol & 41.88 & 0.24 \\
MOL004355 & Spinasterol & 42.98 & 0.76 \\
MOL000449 & Stigmasterol & 43.83 & 0.76 \\
MOL000785 & palmatine & 64.6 & 0.65 \\
MOL000085 & Beta-daucosterol_qt & 36.91 & 0.75 \\
MOL000098 & quercetin & 46.43 & 0.28 \\
\hline & BOL012542 & 0.82 \\
& bidentatoside,ii_qt & 0.59 \\
\hline
\end{tabular}

Table 2 The docking results of the 3 core components and the target protein 
Componds

structure

MAPK1 $/ \mathrm{kal} \cdot \mathrm{mol}-1$

ol-1

ol-1

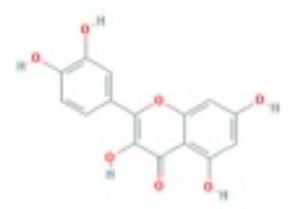

quercetin

$-8.5$

$-8.2$

$-7.7$

wogonin

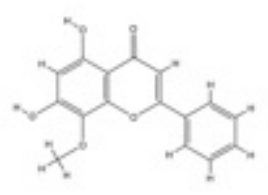

$-8.1$

$-8.7$

$-8.2$

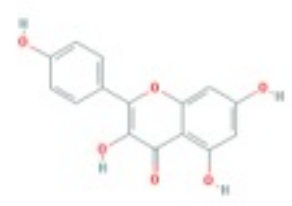

kaempferol

$-8.3$

$-8.2$

$-7.5$

\section{Figures}

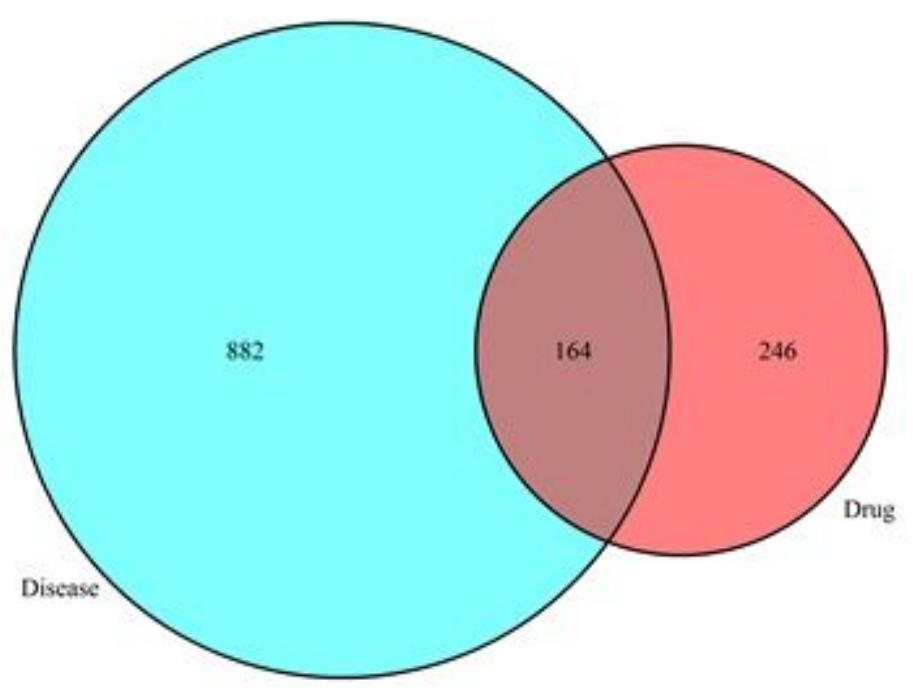


Figure 1

Venn diagram showed 164 potential disease target by the active compound from ABR
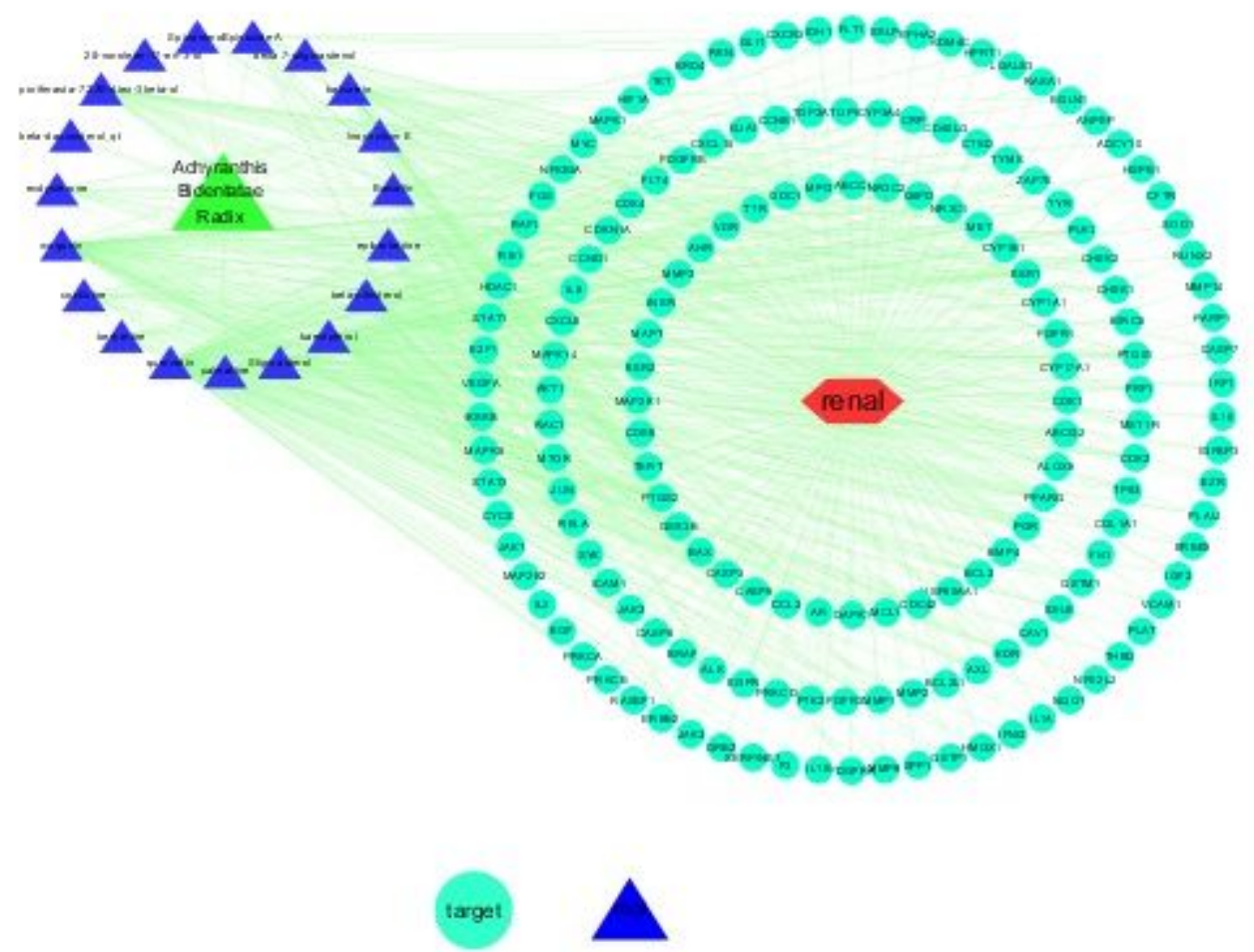

\section{Figure 2}

A medicine-ingredients-targets-disease network with 4 parts; Renal:Renal cell carcinoma;Navy blue:active ingredients of Achyranthis bidentatae radix; blue:164potentia I common targets. 


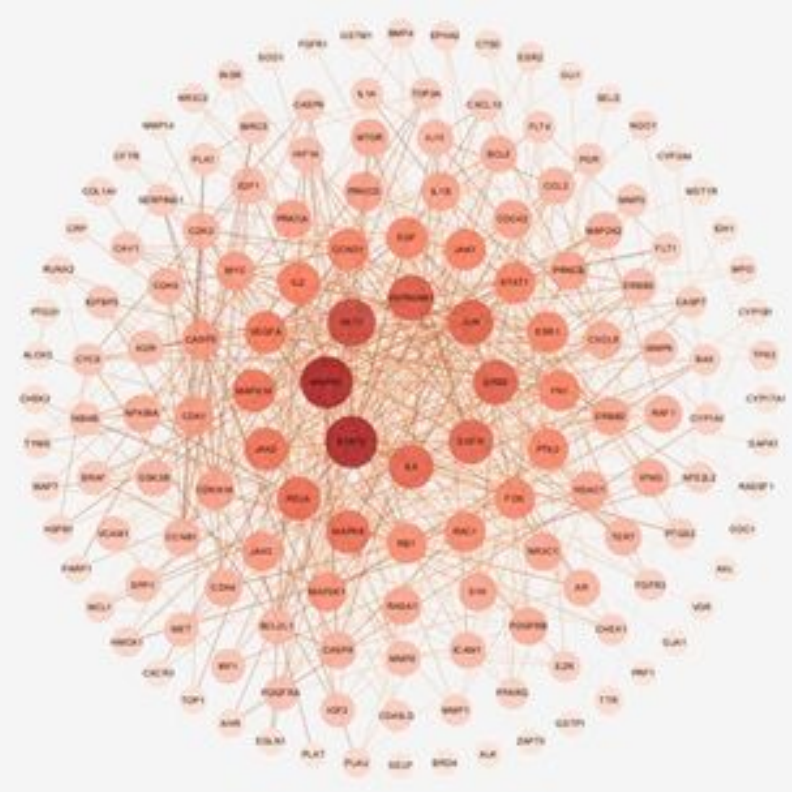

Figure 3

Protein-protein interaction (PPI) network analysis of 164 potential target

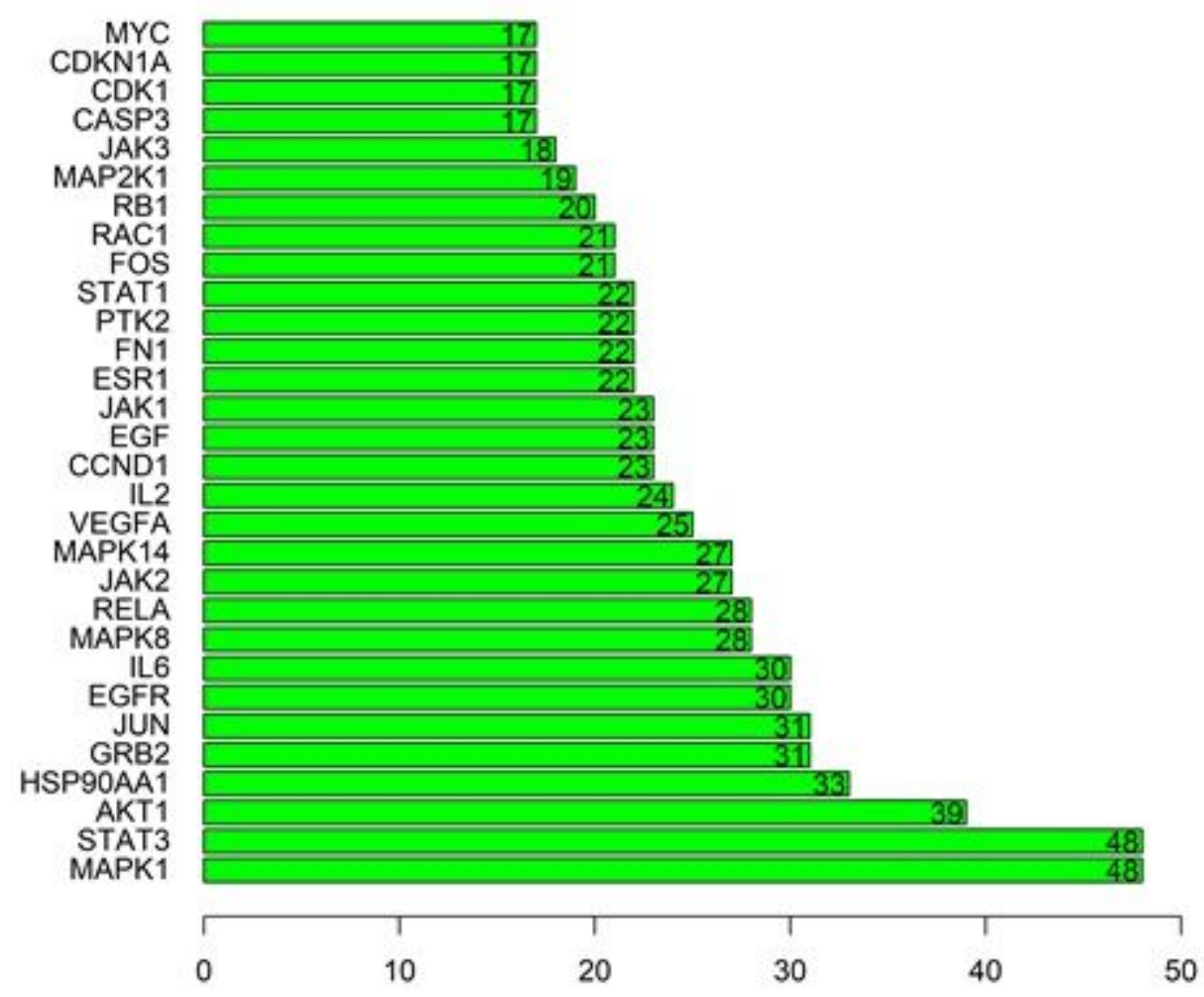

Figure 4

Top 30 targets from protein-protein interaction (PPI) network. 
response to oxidative stress cellular response to oxidative stress

response to reactive oxygen species regulation of reactive oxygen species metabolic process response to antibiotic reactive oxygen species metabolic process epithelial cell proliferation peptidyl-tyrosine phosphorylation peptidyl-tyrosine modificationreproductive structure development reproductive system development extrinsic apoptotic signaling pathway response to lipopolysaccharide response to molecule of bacterial origin regulation of apoptotic signaling pathway aging gland development muscle cell proliferation positive regulation of reactive oxygen species metabolic process

regulation of epithelial cell proliferation -

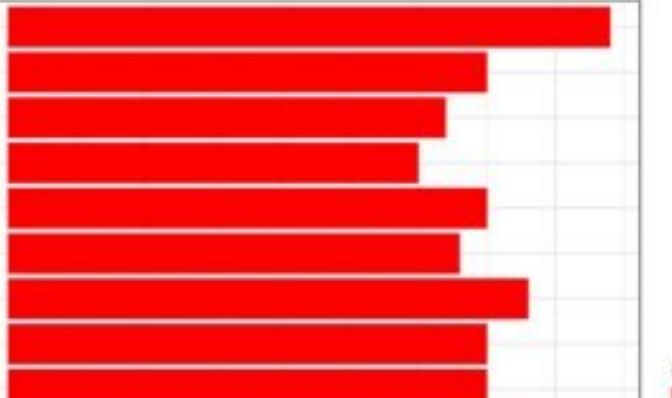

p.adjust
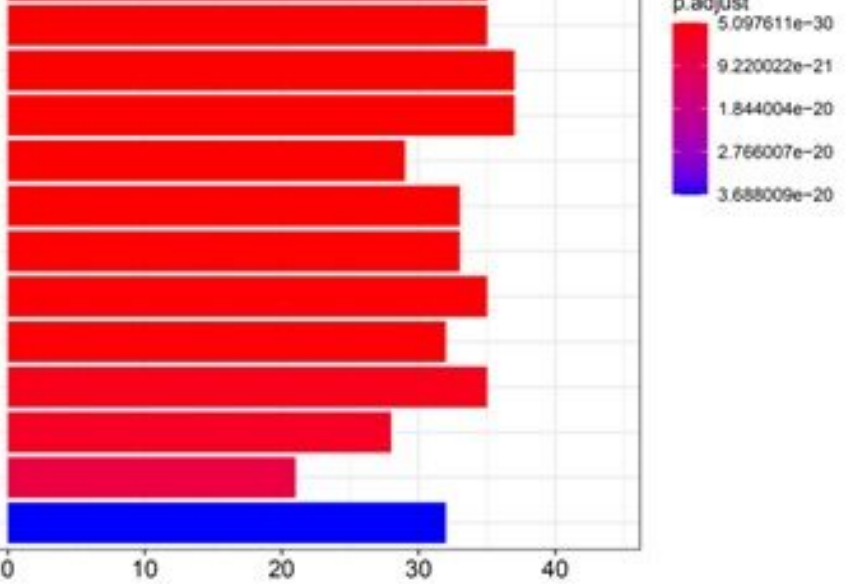

$3.6400000-20$

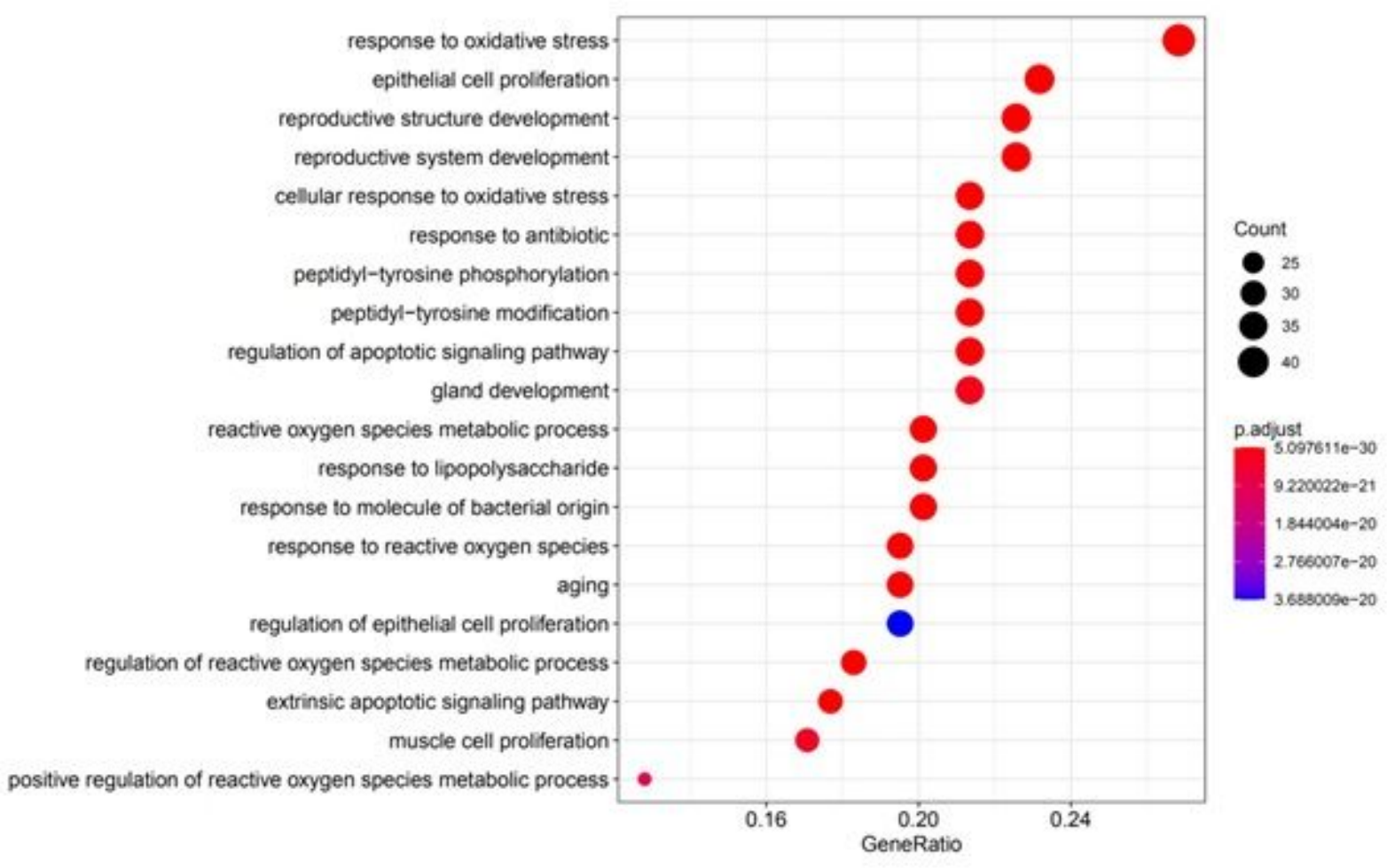

Figure 5

Top 20 enriched Gene Ontology (G0) terms selected from 164 common targets on biological process (Padjusted value $\otimes 0.05)$. 

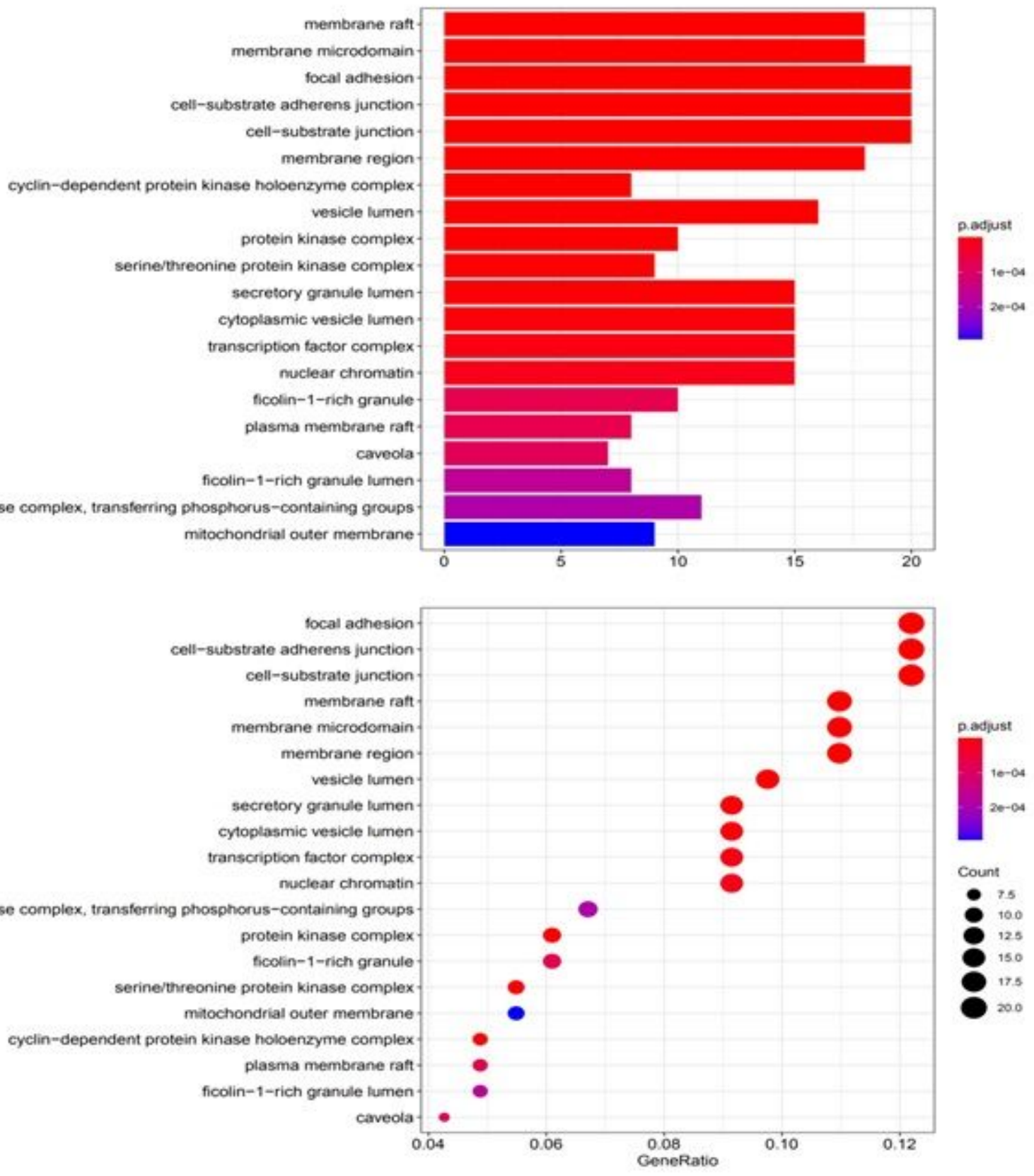

Figure 6

Top 20 enriched Gene Ontology (GO) terms selected from 164 common targets on cell components (Padjusted value $₫ 0.05)$. 
protein tyrosine kinase activity transmembrane receptor protein tyrosine kinase activity transmembrane receptor protein kinase activity phosphatase binding protein phosphatase binding protein serine/threonine kinase activity

cytokine receptor binding histone kinase activity nuclear receptor activity transcription factor activity, direct ligand regulated sequence-specific DNA binding growth factor binding kinase regulator activity ubiquitin-like protein ligase binding integrin binding steroid hormone receptor activity growth factor receptor binding. protein kinase regulator activity cytokine activity hormone receptor binding non-membrane spanning protein tyrosine kinase activity

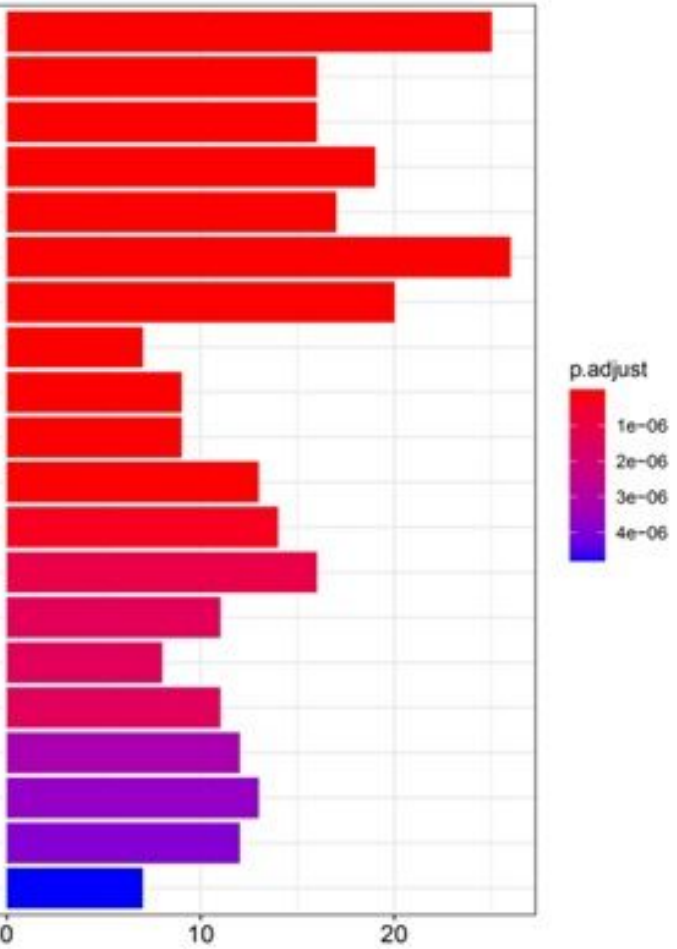
protein serine/threonine kinase activity protein tyrosine kinase activity. cytokine receptor binding phosphatase binding protein phosphatase binding transmembrane receptor protein tyrosine kinase activity transmembrane receptor protein kinase activity ubiquitin-like protein ligase binding kinase regulator activity growth factor binding cytokine activity protein kinase regulator activity hormone receptor binding integrin binding growth factor receptor binding nuclear receptor activity transcription factor activity, direct ligand regulated sequence-specific DNA binding steroid hormone receptor activity. histone kinase activity non-membrane spanning protein tyrosine kinase activity

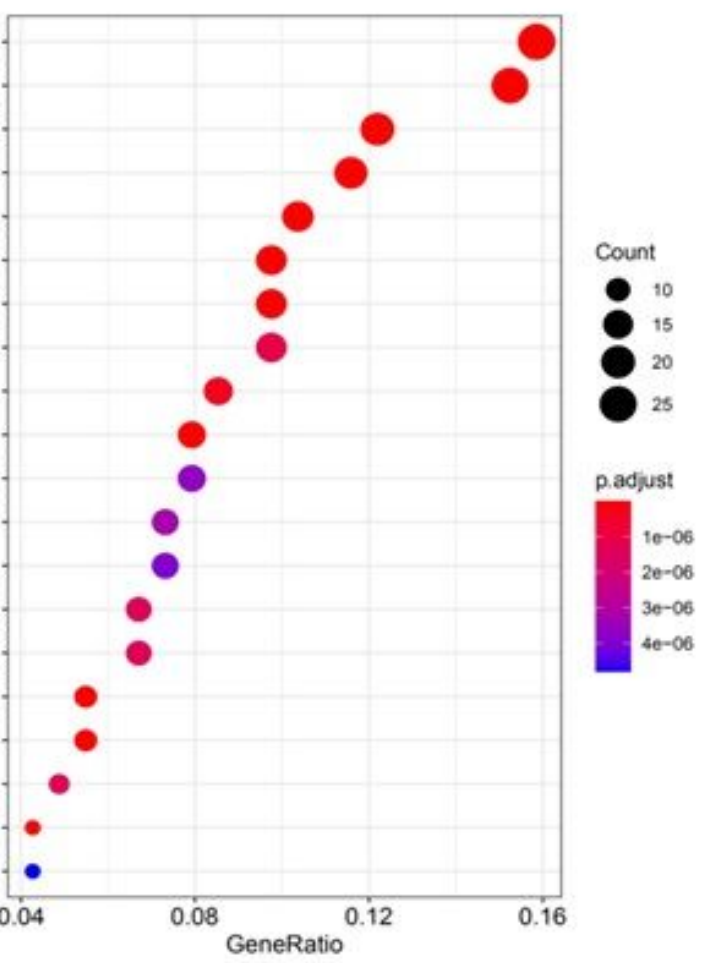

\section{Figure 7}

Top 20 enriched Gene Ontology (GO) terms selected from 164 common targe ts on molecular function(Padjusted value冈0.05). 
AGE-RAGE signaling pathway in diabetic complications

Prostate cancer

EGFR tyrosine kinase inhibitor resistance

PI3K-Akt signaling pathway

Kaposi sarcoma-associated herpesvinus intection-

Hepatis B

Pancreatic cancer-

Bladder cancer

Human cytomegalovirus infection

Non-small cell lung cancer

Proteoglycans in cancer

Fluid shear stress and atherosclerosis

MAPK signaling pathway

Hepatitis C

Endocrine resistance

Colorectal cancer

TNF signaling pathway

Measles

Hepanocellular carcinoma

Epstein-Barr virus infection

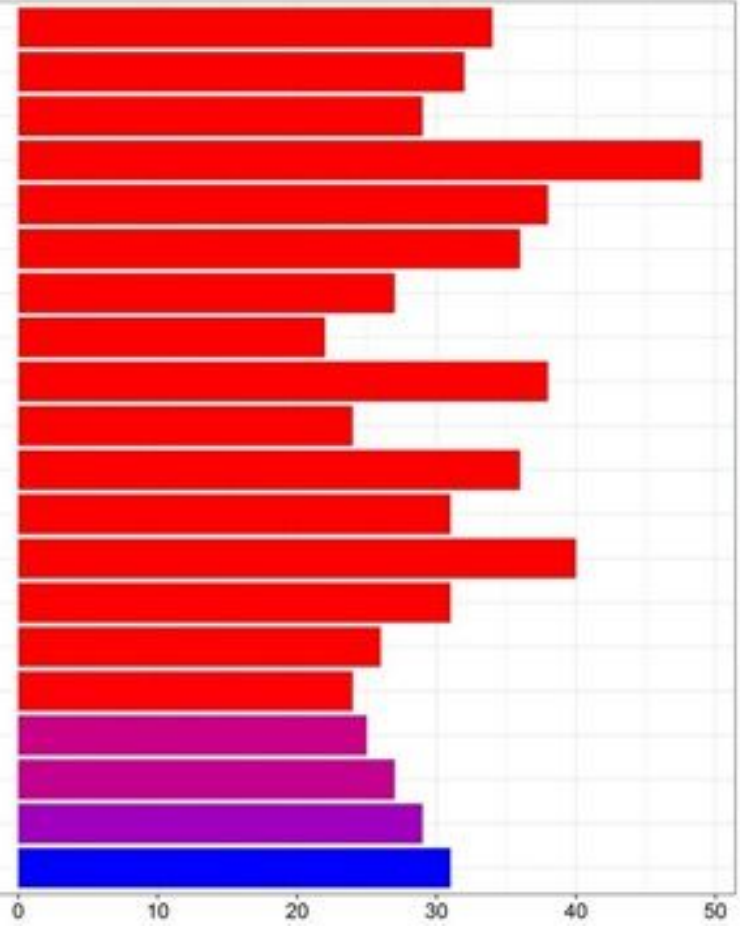

p. adjust

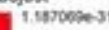

$33012236-19$

secouste-19

s9escebe 19

1.320095e-18

\section{Figure 8}

Top 20 pathways from Kyoto Encyclopedia of Genes and Genomes (KEGG) $₫ \mathrm{P} \llbracket 0.05 \rrbracket$.

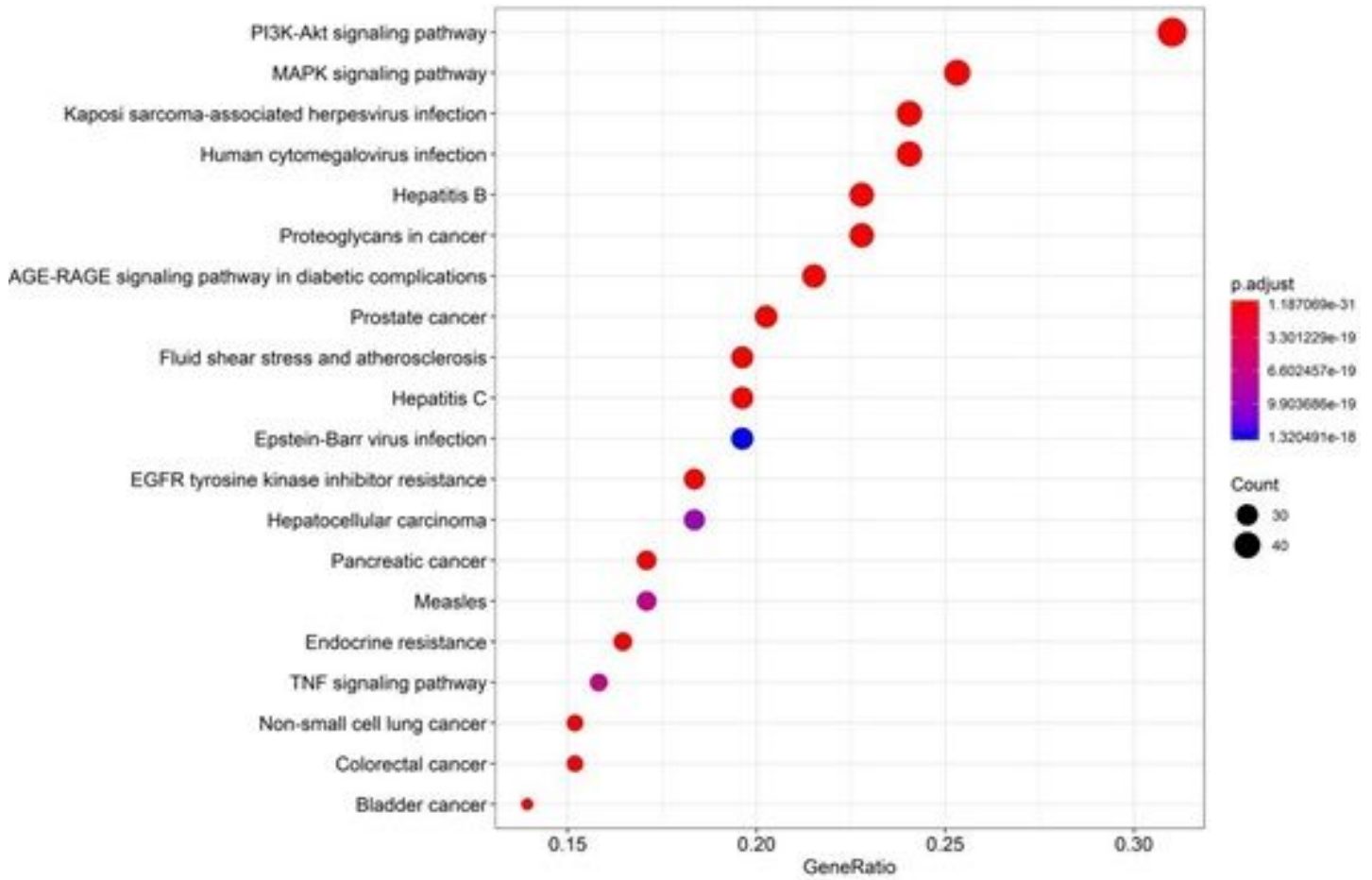

Figure 9

A dot plot to describe P-adjust value range of top 20 pathways. 


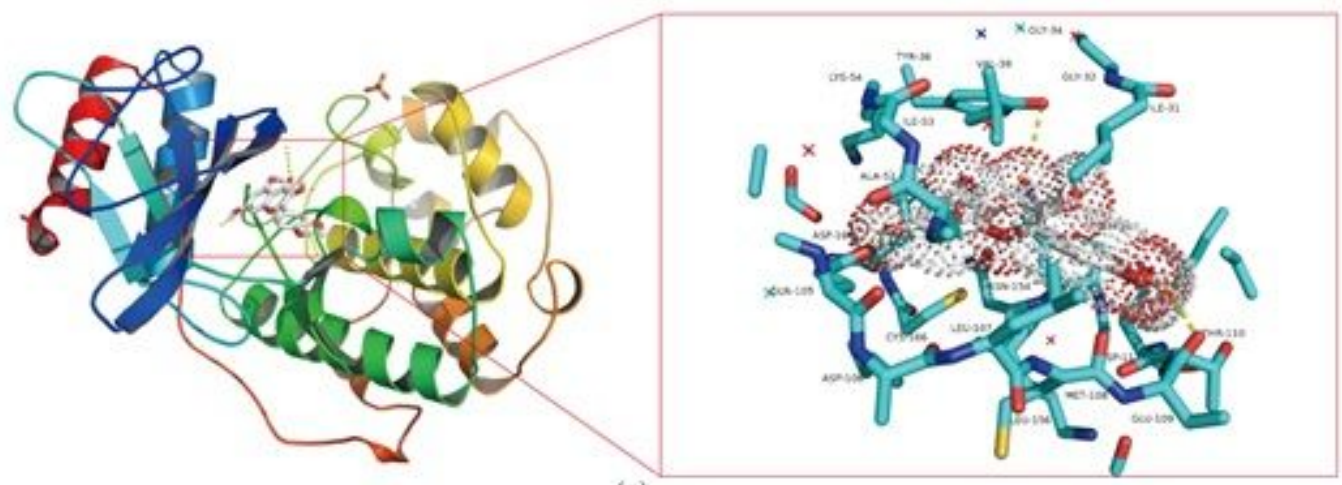

(a)

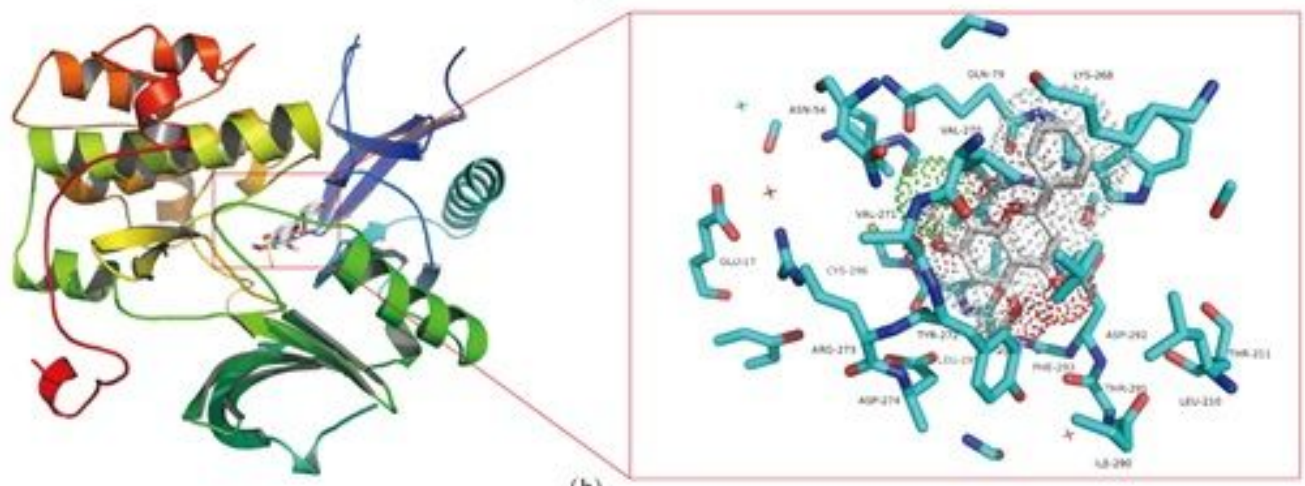

(b)

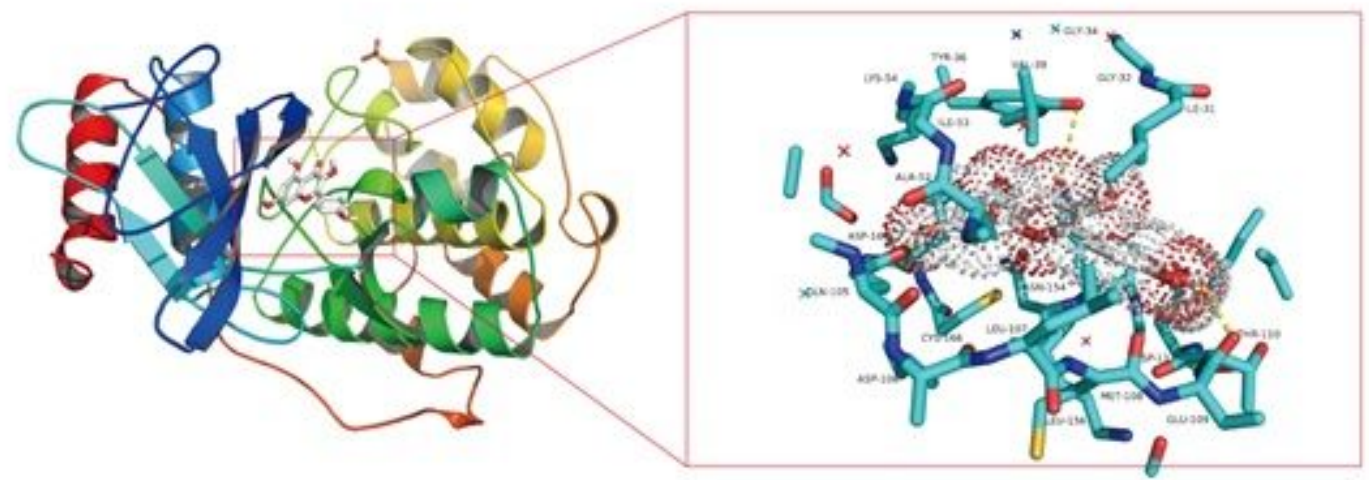

(c)

Figure 10

(a) Quercetin interacts with the target MAPK1 protein (b) Wogonin interacts with the target AKT1 protein.

(C) Kaempferol interacts with the target MAPK1 protein. Different branches of the protein are represented by different colors. Small molecules are represented by Stick and dot. The yellow dashed line represents its hydrogen bond, marking the position of the hydrogen bond and the compound in the compound. 